\title{
The Structure of the Chloroplast considered in Relation to its Function.
}

\author{
BY \\ J. H. PRIESTLEY, B.Sc., \\ Lecturer in Botany, \\ AND \\ ANNIE A. IRVING, \\ Botanical Department, University College, Bristol.
}

With two Figures in the Text.

$\mathrm{O}$

WING to its important rôle in photosynthesis, the structure of the chloroplast has received considerable attention from botanists, and it is interesting to note that a survey of the work done in this direction discloses important points of difference between the various observations made from time to time upon the structure of the chloroplast.

It is clear, however, that the explanation given of the physiological mechanism involved must agree with the results of such structural investigation. Considerations of this kind have led to the publication of the present paper.

\section{HISTORICAL.}

Pringsheim, ${ }^{1}$ after placing green tissues in water at a temperature of $50^{\circ}-60^{\circ} \mathrm{C}$. for $15-60$ minutes, or after exposing them to steam for some time, examined the structure of the chloroplasts microscopically, and came to the conclusion that the ground-substance of the chloroplast is in the form of a hollow sponge-ball, the green colouring matter occurring in the meshes. Having heated the tissues in this manner, he noticed that a colouring matter, which he called hypochlorin, exuded in the form of variously coloured drops.

Kerner ${ }^{2}$ stated that the chlorophyll granules exhibited a pellicle-like thickened outer layer, within which was a colourless porous mass built up of reticular or scaffold-like threads.

1 Pringsheim, Jahrbiicher wissensch. Botan., Bd. xii, I88I.

${ }^{2}$ Kerner, Natural History of Plants, p. 37 I.

[Annals of Botany, Vo1. XXI. No. LXXXIII. July, 1907.] 
The meshes of this sponge-like substance were filled with a green colouring matter dissolved in an oily substance.

Nägeli showed that when chloroplasts were placed in a solution of cane sugar of a certain strength, or in other suitable solutions, they absorbed water, and burst into two valves, which contained the colouring matter.

Timiriazeff ${ }^{1}$ repeated Nägeli's experiments and obtained similar results, using chloroplasts of Phajus on account of their large size. They remained unaltered in sugar solutions of a certain strength, but split as the solution was diluted. He tried to get a clearer idea of their structure by microscopic examination with red light, and thought he could see the green colouring matter arranged around the outer surface of the chloroplast in small granules. These granules appeared as a peripheral ring of black specks.

As a result of the examination of living chloroplasts Wager ${ }^{2}$ came to the conclusion that they consisted of a mass of green granules imbedded in a colourless matrix. In some cases he saw a distinctly fibrillar arrangement of the chlorophyll, and was able to distinguish between a granular structure in the chloroplasts in epistrophe and a fibrillar structure in apostrophe.

His general conclusion was that the chloroplast consisted of a colourless ground substance, of a delicate alveolate structure, in which the chlorophyll was more or less uniformly distributed.

This very scanty historical review ${ }^{3}$ suffices to bring out certain points of agreement and disagreement.

It is almost universally accepted that the chloroplasts consist of a colourless ground substance, presumably proteid in nature, and that the chlorophyll is in some way distributed within this substance. Probably it is held in solution in some vegetable fat or oil.

A point upon which there is disagreement is the distribution of the chlorophyll in the granule.

Some observers state that it occurs in a ring at the periphery of the plastid, others that it is diffused uniformly throughout.

It is evident that this question affects the validity of the observations of Nägeli and Timiriazeff upon the splitting of the granule.

As Timiriazeff ${ }^{4}$ has previously shown, the distribution of the chlorophyll may be of considerable importance from a physiological point of view. The energy transformation taking place in the chloroplast, apparently requires that the chlorophyll, absorbing the energy, should be distributed

1 Timiriazeff, Croonian Lecture, Proceedings of Royal Society, vol. lxxii.

2 Wager, Presidential Address to Section K, British Association Meeting in South Africa, I905.

3 For a fuller historical account see Czapek, Biochemie, vol. i, p. 445.

- Timiriazeff, loc. cit., p. 457. 
in a very thin layer. Then the thinner the layer in which a definite amount of chlorophyll is disposed, the greater the amount of energy set free at any part of that layer. Consequently the efficiency of the chloroplast in bringing about the photolytic decomposition of carbon dioxide must depend on the thickness of the layer.

As some recent work carried out by one of us, in conjunction with Mr. Usher, ${ }^{1}$ had confirmed the impression that the thickness of the layer was of importance, it seemed desirable to repeat some of these previous observations, and to see if it were possible to come to an independent conclusion.

\section{The Microscopic Structure of the Chloroplast.}

Plants containing relatively large chloroplasts, generally either Selaginella Martensii, S. Kraussiana, or Chlorophytum elatum, were taken, and various attempts were made to elucidate their structure.

In the case of the Selaginella, it was difficult even when using Zeiss

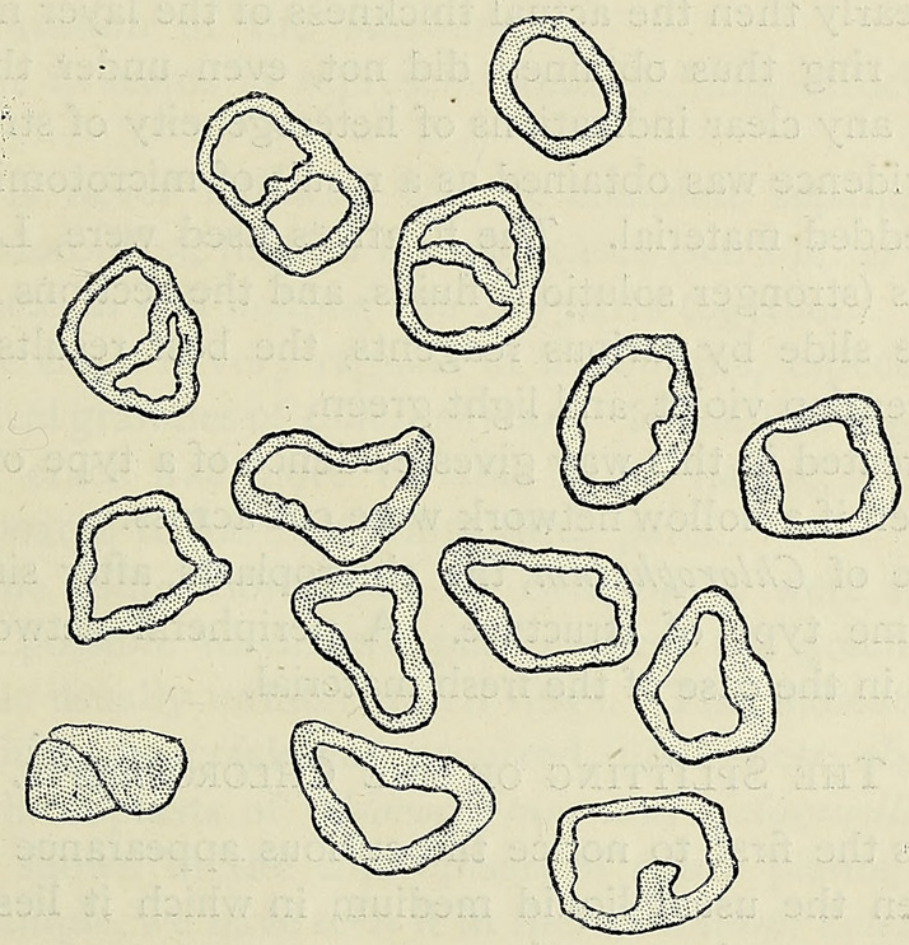

FIG. I.

apochromatic immersion objectives $\left(\frac{1}{8}\right.$ and $\left.\frac{1}{12}\right)$ and an achromatic condenser, to discover any signs of heterogeneity of structure; but with Chlorophytum such indications were very marked. The chloroplast of the latter plant clearly seemed to consist of a network with chlorophyll in the meshes. Furthermore, in many cases it was possible to obtain optical

\footnotetext{
1 Usher and Priestley, Proceedings of the Royal Society B, vol. lxxviii, I 906.
} 
sections of the chloroplasts, which indicated the existence of a distinct peripheral layer to this network. This layer contained the colouring matter.

To obtain chloroplasts for observation in these cases, the leaves of the plant were simply crushed, and some of the green mass was mounted in its own sap.

As the chloroplasts are unusually large in the case of Selaginella, the diameter of the largest being about $0.02 \mathrm{~mm}$., an attempt was made to obtain sections of the chloroplast with the fresh material. The sections of the chloroplasts shown in Fig. I were obtained in the following way :The chloroplasts were expresssd in the juice of the plant, mixed with gum and glycerine, frozen, and cut to the thickness of I $\mu$ by means of a Reichert sliding microtome. A previous attempt to obtain sections from material imbedded in gelatine and frozen proved unsuccessful. The result of this experiment leaves no doubt in our minds, that, in these causes, the chlorophyll is restricted to the outer layer of the chloroplast. This outer layer has a thickness varying approximately from $0.003 \mathrm{~mm}$. to $0.00 \mathrm{Imm}$, as seen in section. Clearly then the actual thickness of the layer must be less.

The green ring thus obtained did not, even under the $\frac{1}{12}$ inch apochromatic, give any clear indications of heterogeneity of structure.

Further evidence was obtained as a result of microtoming the properly fixed and imbedded material. The fixatives used were, Lang's, Merkel's, and Flemming's (stronger solution) fluids, and the sections were afterwards strained on the slide by various reagents, the best results being obtained by the use of gentian violet, and light green.

Material treated in this way gives evidence of a type of structure such as would be seen if a hollow network were cut across.

In the case of Chlorophytum, the chloroplasts, after similar treatment, showed the same type of structure. A peripheral network was clearly indicated, even in the case of the fresh material.

\section{The Splitting of the Chloroplasts.}

Nägeli was the first to notice the curious appearance obtained in the chloroplast when the usual liquid medium in which it lies is replaced by one of a different osmotic strength.

Timiriazeff has since confirmed these observations, which, however, have not received much attention from other workers upon the subject.

We have also repeated Nägeli's experiments, and have obtained confirmatory results.

The granules, when extracted in their own sap, show no signs of such a crack. But upon adding water, or dilute sugar solution, whilst the chloroplasts still remained under observation, they invariably split, showing, in consequence, a fine dark or light line, which, in a very large percentage 
of the chloroplasts, was medianly situated (Fig. 2). The crack, of course, is only visible in certain planes, and as some of the chloroplasts rolled over, it was possible to see it appear and disappear.

A similar crack could be obtained by irrigation with dilute salt solution. But these cracks never appeared in concentrated solutions of either salt or sugar.

When the concentrated salt or sugar solution in which the granules are lying is gradually diluted, at a certain degree of dilution the split rapidly appears in a number of the granules. In most cases it appears simultaneously across the whole width of the granule, but in one or two cases it was seen first at one side, afterwards extending rapidly across the chloroplast.

When the split has once appeared, it is impossible to make it disappear by increas-

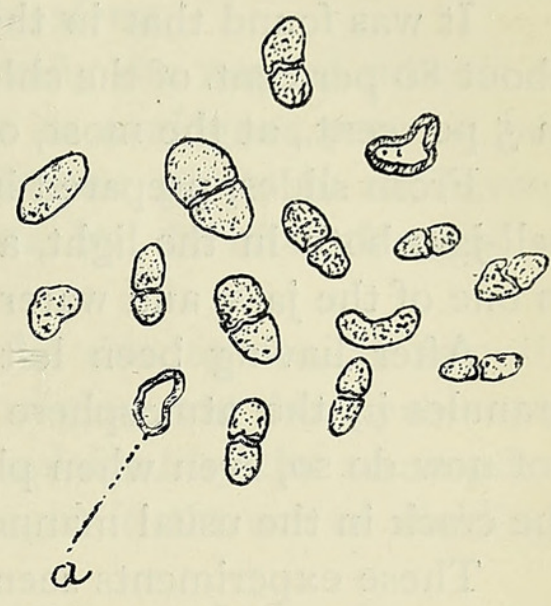

FIG. 2. ing the concentration of the surrounding solution, so that it seems to represent some definite structural change in the granule.

The split is never followed by the complete separation of the two halves of the chloroplast, though in some cases (see Fig. 2) it is impossible to see any connexion still holding the two parts together.

Usually the crack is very regular in its outline, especially in the case of the symmetrical granules of Chlorophytum. In the less regular granules of Selaginella, the crack was more variable in its nature, and in some cases the granule appeared to have split into three parts.

Some of the split chloroplasts of Selaginella were frozen and cut. In these it was possible to discern places where the green layer was discontinuous in the usually uninterrupted rings. This indicated, presumably, the region in which the crack had occurred. All these observations were made on the chloroplasts of Chlorophytum and Selaginella, but the split can readily be shown in the chloroplasts of many plants, when similarly treated; for example, we have seen it in the Gevanium, Coleus, Elodea, Iris, and Amaryllis.

\section{Effect of Light and Carbon Dioxide on the Splitting.}

It was thought that even after expression in sap, the chloroplasts might still be able to form sugar. If this sugar were still retained within the chloroplast there should be a difference of osmotic pressure, comparable to that previously obtained by diluting the surrounding medium, and under the circumstances, a crack might be expected to appear. Leaves of S. Martensii and Chlorophytum were placed in water in a dark cupboard 


\section{I 2 Priestly and Irving.-The Structure of the Chloroplast}

for three days; the granules were then extracted under pressure, and upon examination they showed no split. Drops of sap containing the chloroplasts were then mounted on microscope slides in the ordinary way; and all the slides were placed in a moist atmosphere in the greenhouse, some being kept in a sunny position, others in the dark.

It was found that in the slides which had been placed in the sunlight, about 80 per cent. of the chloroplasts had split, whereas a crack had appeared in 5 per cent., at the most, of those kept in the dark.

Fresh slides, prepared in the same way, were then mounted under two bell-jars both in the light, a beaker containing caustic potash being placed in one of the jars, and water in the other.

After having been left for a week, it was found that none of the granules in the atmosphere free from carbon dioxide had split, and would not now do so, even when placed in distilled water. The other set showed the crack in the usual manner.

These experiments seem to indicate that when the granules are isolated in this manner, they are still alive, and capable of synthesizing sugar from carbon dioxide, and water, in the presence of light. The sugar thus formed collects within the granule, while the outside medium remains at its former osmotic strength, and consequently, a split occurs just as when the sap is diluted.

\section{The Effect of Chloroform.}

Leaves of Selaginella and Chlorophytum were killed by treatment with chloroform vapour for two hours. The juice was then expressed as before, and the chloroplasts mounted in distilled water.

In this case it was quite impossible to see any split in the granules. By placing them in a dilute solution of eosin, it was seen that the outer membrane of the plastid had been rendered completely permeable by the action of the poison, the eosin passing into the granule, and colouring it, but not concentrating within it, as it does when the chloroplast is living.

\section{The Nature of the Split.}

This split then can be brought about in the chloroplast either by decreasing the concentration of the surrounding sap, or by increasing the concentration of the sugar solution within the granules.

It seems, therefore, to be the result of an internal swelling caused by water passing into the chloroplast, and of the consequent separation of the outer peripheral ring into two halves, the inner colourless stroma not dividing, but holding the two together.

The passage of water inwards may be due to a difference of osmotic pressure on either side of the peripheral layer, which functions as a semi- 
permeable membrane, though if this is the case in the living cell, it is difficult to see how the sugar formed by the chloroplast is passed out into the surrounding cytoplasm.

On the other hand, the absorption of water may be due to imbibition by the internal colourless stroma. One would expect neither of these processes to take place after treatment with chloroform.

It has been suggested that this split is really the normal process of division in the chloroplast. It may certainly be an analogous process, but its rare occurrence, except under certain conditions, points to its having been artificially induced in these experiments by definite changes of environment.

Normal division of the chloroplast may be due to similar osmotic differences existing between cytoplasm and chloroplast, but the appearances noted in our experiments, certainly cannot be explained by the assumption that a greater number of chloroplasts were caught in the act of dividing, in one case than in the other.

\section{SUMMARY.}

By microscopic investigation of living and fixed material, we have come to the conclusion that in the large chloroplasts of Chlorophytum elatum, Selaginella Martensii, and S. Kranssiana, the chlorophyll is restricted to the peripheral ring of the chloroplast, where it is held in the meshes of a network.

This is in agreement with the views of Timiriazeff concerning the function of chlorophyll in absorbing the radiant energy necessary for the photolytic decomposition of carbon dioxide.

Our confirmation of the observations of Nägeli and Timiriazeff in regard to the splitting of the chloroplasts when placed in a solution of small osmotic strength, is also in agreement with this view of their structure. 


\section{$2 \mathrm{BHL}$ Biodiversity Heritage Library}

Priestley, J.H. and Irving, Annie A. 1907. "The structure of the chloroplast considered in relation to its function." Annals of botany 21, 407-413. https://doi.org/10.1093/oxfordjournals.aob.a089143.

View This Item Online: https://www.biodiversitylibrary.org/item/235747

DOI: https://doi.org/10.1093/oxfordjournals.aob.a089143

Permalink: https://www.biodiversitylibrary.org/partpdf/318882

\section{Holding Institution}

Smithsonian Libraries

\section{Sponsored by}

Biodiversity Heritage Library

\section{Copyright \& Reuse}

Copyright Status: Not in copyright. The BHL knows of no copyright restrictions on this item.

This document was created from content at the Biodiversity Heritage Library, the world's largest open access digital library for biodiversity literature and archives. Visit BHL at https://www.biodiversitylibrary.org. 